\title{
A Case of VACTERL Association Complicated with Multiple Rib Abnormalities
}

\author{
Zeng Zhiguo, Zhang Guowei, Ji Zhisheng, Yang Yuhao, Yang Hua, Lin Hongsheng*
}

Department of Orthopedics, The First Affiliated Hospital of Jinan University, Jinan, China

Email address:

tlinhs@jnu.edu.cn (Lin Hongsheng)

${ }^{*}$ Corresponding author

\section{To cite this article:}

Zeng Zhiguo, Zhang Guowei, Ji Zhisheng, Yang Yuhao, Yang Hua, Lin Hongsheng. A Case of VACTERL Association Complicated with Multiple Rib Abnormalities. Clinical Neurology and Neuroscience. Vol. 4, No. 1, 2020, pp. 1-4. doi: 10.11648/j.cnn.20200401.11

Received: December 17, 2019; Accepted: December 27, 2019; Published: February 4, 2020

\begin{abstract}
VACTERL association is an acronym that includes vertebral anomalies (V), anal atresia (A), cardiac defects (C), tracheoesophageal fistula (TEF) or esophageal atresia (EA), renal anomalies (R), and limb defects (L). Rib abnormalities have rarely been reported with the VACTERL association. The second case of VACTERL association complicated with multiple rib abnormalities will be reported in this case report. A 4-year-old girl who had been diagnosed with congenital cardiovascular disease and received surgical corrections soon after birth consulted our institution by complaining congenital scoliosis. The little girl was diagnosed with VACTERL association (congenital cardiovascular disease, scoliosis with hemivertebra and butterfly vertebra, and nephrolithiasis) and congenital multiple rib abnormalities. The Cobb angle of the main curve was $29.3^{\circ}$ before surgery, $19.9^{\circ}$ after surgery, and $23^{\circ}$ at last follow-up. Multiple rib abnormalities may be seen in the VACTERL association. Clinicians should have a high index of suspicion when evaluating patients with rib abnormalities associated with VACTERL. It is extremely necessary for careful physical examination and detailed auxiliary examination to each system (including echocardiography, computed tomography, magnetic resonance imaging, and so on) when diagnosing VACTERL association. Also, recognizing and understanding the congenital multiple system malformation is important, which aids in the diagnosis of disease and accordingly plan the therapeutic interventions. Early diagnosis of congenital scoliosis with appropriate surgical intervention decreases long-term morbidity.
\end{abstract}

Keywords: VACTERL Association, Rare Diseases, Rib Abnormalities

\section{Introduction}

VACTERL association is a rare congenital multiple system malformation. VACTERL association is typically defined by the presence of at least three of the following congenital malformations: vertebral defects, anal atresia, cardiac defects, tracheoesophageal fistula, renal anomalies, and limb abnormalities. In addition to these core component feature, patients may also have other congenital anomalies. The condition is ascertained clinically by the presence of the above-mentioned malformations; importantly, there should be no clinical or laboratory-based evidence for the presence of one of the many similar conditions, as the differential diagnosis is relatively large. Although diagnostic criteria vary, the incidence is estimated at approximately 1 in 10,000 to 1 in 40,000 live-born infants [1]. There is overall no strong evidence for an increased incidence of VACTERL association in certain areas of the world or in specific ethnic populations. Relatively large cohorts of patients have been described from all parts of the world in which such studies have been conducted. Rib abnormalities have rarely been reported with the VACTERL association. We present a patient with VACTERL association found to have multiple rib abnormalities. And the patient has received surgical corrections of cardiovascular malformation and spinal hemivertebra deformity resection.

\section{Case Presentation}

The patient is a 4 years old girl. She was a twin baby girl at birth. After birth, she showed clinical symptoms such as dyspnea, heart failure, and cyanosis. Her mother denied any history of radioactive, toxic substances, or drug exposures during her pregnancy. Echocardiography found persistent 
truncus arteriosus (PTA), ventricular septal defect (VSD), atrial septal defect (ASD), and pulmonary hypertension. She was diagnosed as PTA, VSD, and ASD, and received surgical corrections of total arterial stem correction, ventricular septal defect repair, and atrial septal defect repair soon after birth. Renal ultrasound examination: nephrolithiasis. A spinal radiograph showed rib anomalies and thoracic vertebral anomalies. One of the twin girls did not find any physical abnormalities. As the girl had renal anomalies, rib anomalies, thoracic vertebral anomalies, and cardiovascular anomalies, the VACTERL association was diagnosed. Spinal CT suggestion: Scoliosis deformity, T8, T10, T11, T12, L3 vertebral developmental malformations, most of which are

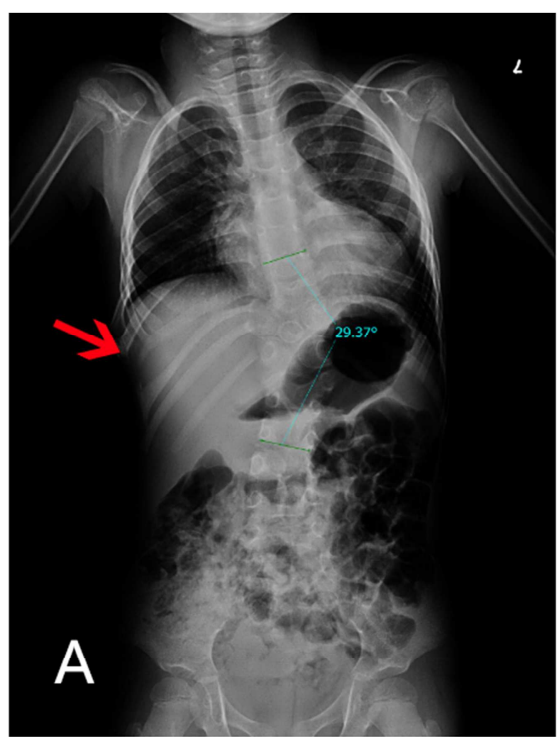

butterfly vertebral changes, some of which are hemivertebra changes, and the vertebral arches on both sides of T12 vertebra are not closed. Posterior T12 hemivertebra resection and short segment fusion with pedicle screw fixation in our hospital 2018 year. The Cobb angle of the main curve was $29.3^{\circ}$ before surgery, $19.9^{\circ}$ after surgery, and $23^{\circ}$ at last follow-up, multiple rib deformities on the right. The patient's congenital scoliosis deformity corrected and was discharged 14 days later. The patient was followed up for 1 year. As the time of writing, the patient has remained well and no longer-term sequelae and recurrent. The routine follow-up examinations among blood and urine testing, spinal X-rays, echocardiography and renal function are normal.
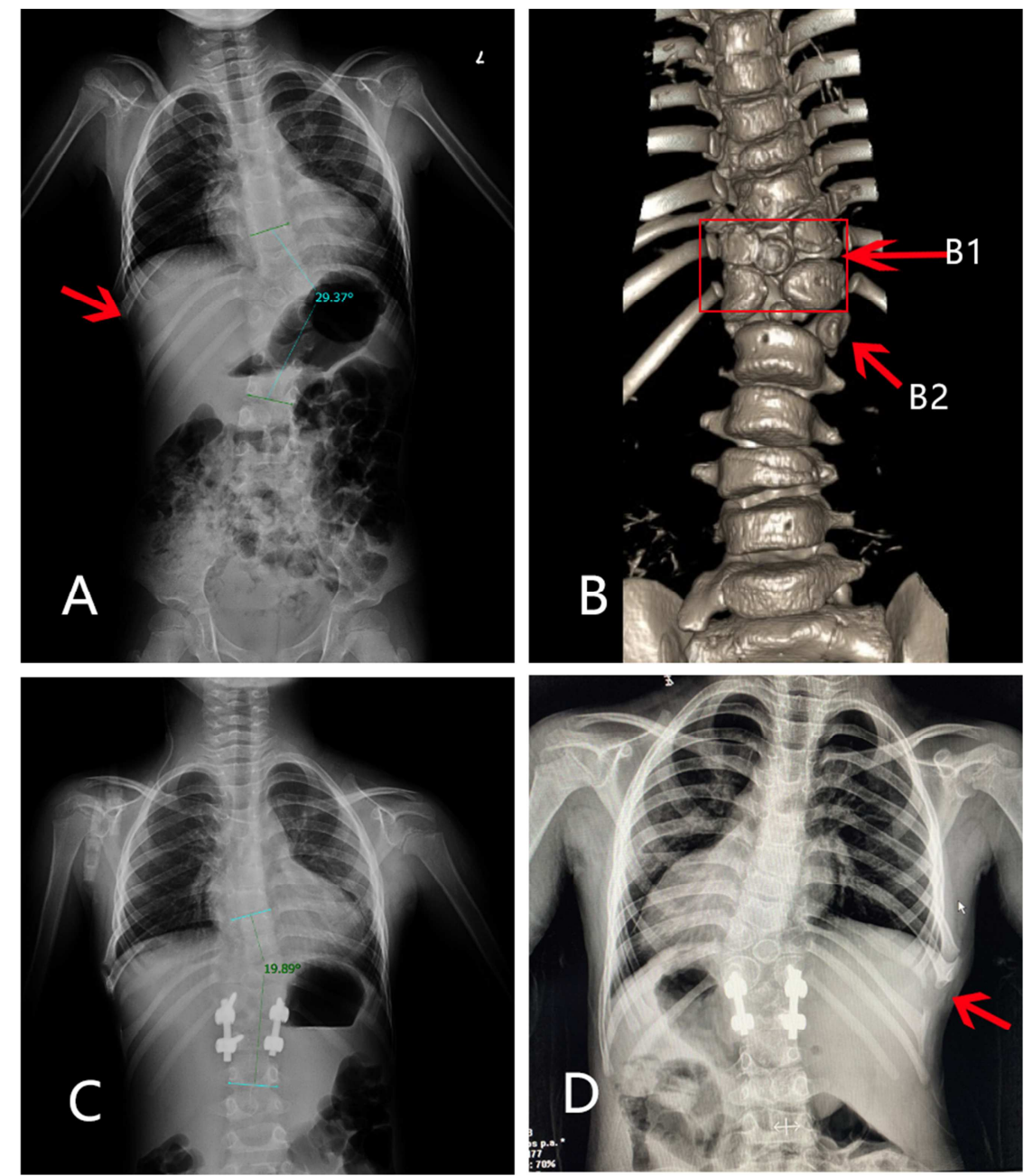

Figure 1. Preoperative and postoperative imaging examination of the patient. A, preoperative X-ray films showed rib anomalies on the right, scoliosis deformity and thoracic vertebral anomalies. B, Preoperative spinal CT suggestion: Scoliosis deformity, vertebral developmental malformations, most of which are butterfly vertebral changes (B1, red box and red arrow), T12 hemivertebra changes (B2, red arrow). $C$ and $D$, postoperatively and follow-up $X$-ray showed that scoliosis has been corrected to some extent, T12 hemivertebra has been resected and rib anomalies on the right still remains.

\section{Discussion}

VACTERL association is a rare and complex condition with highly heterogeneous etiology and manifestations [2]. Approximately $90 \%$ of VACTERL association cases occur sporadically [3], with an empiric recurrence risk of $1 \%$ or less [4]. VACTERL association has previously been reported to coexist with the absence of the one rib. Most doctors require at least 3 component features for diagnosis, without clinical or laboratory-based evidence of the overlapping conditions, while others emphasize the presence of certain component features, especially TEF or anorectal malformation. No definite etiology and pathogenesis has been proven, but a defect in mesodermal differentiation due to a variety of causes (genetic, environmental and multi-factors are implicated), in early first trimester (between 6th and 10th weeks of gestation), 
has been suggested $[5,6]$.

Patients and families with features of VACTERL association are told very little about long-term prognoses and outcomes, perhaps due to a dearth of published information [7], so the management of patients is complex. According to each component feature of VACTERL association, treatment involves surgical correction of the congenital abnormality and the long-term management $[1$, 7]. Most patients with congenital scoliosis need surgery to avoid severe spinal curvature and imbalance when the bones mature. The treatment is different from idiopathic scoliosis as long as possible to delay the fusion time. Congenital scoliosis should be operated as early as possible during its progression to correct deformities and prevent its progression into structural spinal decompensation. Untreated hemivertebra, both full or semisegmented, can lead to serious spinal deformities. It is difficult to predict the natural course of hemivertebrae $[8,9]$.

In the case of progressive deformity, nonsurgical methods such as bracing cannot prevent deformity progression. There is an agreement in the literature on the application of surgical treatment in progressive curves $[10,11]$. In a multicentric study, it compared hemiepiphysiodesis or in situ fusion, instrumented fusion without hemivertebra excision, and instrumented hemivertebra excision. They found that although a higher rate of complications was seen with hemivertebra resection compared to the other two methods, better correction rates were achieved [12]. Therefore, hemivertebra excision is the only method that allows complete correction of the deformity by elimination of the pathology and yields predictable results [13]. Ruf reported that 28 cases of congenital scoliosis with an average age of 3 years and 4 months were treated with posterior hemivertebra resection, and the operation achieved good results. Correction surgery of congenital scoliosis should be performed early, before the development of severe local deformities and secondary structural changes, especially in patients with expected deterioration. Posterior resection of the hemivertebra with transpedicular instrumentation allows for early intervention in very young children. Excellent correction in the frontal and sagittal planes and a short segment of fusion allows for normal growth in the unaffected parts of the spine [14]. This patient is 4 years old, has a good spine flexibility, a small vertebral body, and a soft texture. The Risser sign is grade 0 . The skeletal development is still immature and the deformity may be further aggravated. Early surgery is needed. Previous research shows that if rapid progression is documented or expected, surgical intervention as early as possible is warranted to prevent secondary structural changes. The surgical treatment should be focused on and limited to the site of malformation. The aim of surgery is the correction of the deformity at the site of asymmetrical growth. This can be achieved either by resection of a hemivertebra or by performing a vertebral column resection or other type of osteotomy [15].

In patients with a single hemivertebra malformation, the progress of the malformation is often easy to judge, and the treatment plan is clearer. Posterior hemivertebra resection and monosegmental fusion seems to be an effective approach for the treatment of congenital scoliosis caused by hemivertebra, allowing for excellent correction in both the frontal and sagittal planes [16]. However, for patients with multiple hemivertebra deformities, the spinal deformity caused by the interaction of asymmetrical loads in different parts and the variability of local deformities is extremely complicated. With the natural growth of the spine, the progress of its deformities is also difficult to predict, and the choice of clinical treatment options is also a test.

The management of patients with VACTERL/VATER association typically centers around the surgical correction of the specific congenital anomalies (typically anal atresia, certain types of cardiac malformations, and/or tracheoesophageal fistula) in the immediate postnatal period, followed by long-term medical management of sequelae of the congenital malformations. If optimal surgical correction is achievable, the prognosis can be relatively positive, though some patients will continue to be affected by their congenital malformations throughout life. Importantly, patients with VACTERL association do not tend to have neurocognitive impairment. [1]

Once a newborn with a congenital malformation in one system is found clinically, it is necessary to be vigilant about the presence of congenital malformations in other systems, and comprehensive screening must be carried out, especially the esophagus, anus, vertebra, cardiovascular, urogenital system, and limbs, found three or more related abnormalities, neonatal VACTERL association can be diagnosed. The number of reports of neonatal VACTERL association is rare, and it is estimated that it is related to the lack of awareness and attention of medical staff. Another possible reason is that the diagnostic level of prenatal ultrasound examination is increased, and abortion or induction of labor after termination of multiple abnormalities is found, leading to a decrease in neonatal VACTERL association.

\section{Conclusion}

Multiple rib abnormalities may be seen in VACTERL association. Clinicians should have a high index of suspicion when evaluating patients with rib abnormalities associated with VACTERL. It is extremely necessary for careful physical examination and detailed auxiliary examination to each system (including echocardiography, computed tomography, magnetic resonance imaging, and so on) when diagnosing VACTERL association. Also, recognizing and understanding the congenital multiple system malformation is important, which aids in the diagnosis of disease and accordingly plan the therapeutic interventions. Early diagnosis of congenital scoliosis with appropriate surgical intervention decreases long-term morbidity.

\section{Conflict of Interests}

The authors have no conflicts of interest to disclose. 


\section{Acknowledgements}

This work was supported by National Natural Science Foundation of China (81771331), Natural Science Foundation of Guangdong Province, China (2017A030313595), and Guangzhou science and technology project (201605130849016).

\section{References}

[1] Solomon BD. VACTERL/VATER association. Orphanet J Rare Dis. 2011. 6: 56.

[2] Chen Y, Liu Z, Chen J, et al. The genetic landscape and clinical implications of vertebral anomalies in VACTERL association. J Med Genet. 2016. 53: 4317.

[3] Yang L, Li S, Zhong L, et al. VACTERL association complicated with multiple airway abnormalities: A case report. Medicine 2019, 98 (42): e17413.

[4] Nezarati M, McLeod D. VACTERL manifestations in two generations of a family. Am J Med Genet. 1999. 82: 402.

[5] Xu GQ, Zhou QC, Zhang M, et al. TACRD and VACTERL associations in a fetus: case report and review of the literature. Int J Pediatr Otorhinolaryngol. 2013. 77: 20815.

[6] Behera B, Shaw S, Raj R. Vacterls association with pulmonary arterial hypertension and least vertebral anomaly. YUVA J Med Sci. 2015. 1: 2830.

[7] Raam MS, Pineda-Alvarez DE, Hadley DW, et al. Long-term outcomes of adults with features of VACTERL association. Eur J Med Genet. 2011. 54: 3441.

[8] McMaster M. J., Singh H. Natural History of Congenital
Kyphosis and Kyphoscoliosis. A Study of One Hundred and Twelve Patients. The Journal of Bone \& Joint Surgery. 1999. 81 (10): 1367-1383.

[9] Ruf M., Harms J. Hemivertebra resection by a posterior approach: innovative operative technique and first results. Spine. 2002. 27 (10): 1116-1123.

[10] Holte D. C., Winter R. B., Lonstein J. E., Denis F. Excision of hemivertebrae and wedge resection in the treatment of congenital scoliosis. The Journal of Bone \& Joint Surgery. 1995. 77 (2): 159-171.

[11] Jalanko T., Rintala R., Puisto V., Helenius I. Hemivertebra resection for congenital scoliosis in young children: comparison of clinical, radiographic, and health-related quality of life outcomes between the anteroposterior and posterolateral approaches. Spine. 2011. 36 (1): 41-49.

[12] Yaszay B., O'Brien M., Shufflebarger H. L., et al. Efficacy of hemivertebra resection for congenital scoliosis: a multicenter retrospective comparison of three surgical techniques. Spine. 2011. 36 (24): 2052-2060.

[13] Erturer, R. E., et al. The Results of Hemivertebra Resection by the Posterior Approach in Children with a Mean Follow-Up of Five Years. Adv Orthop. 2017: 4213413.

[14] Ruf, M. and J. Harms. Posterior hemivertebra resection with transpedicular instrumentation: early correction in children aged 1 to 6 years. Spine. 2003. 28 (18): 2132-2138.

[15] Fekete, T. F., et al. Congenital malformations of the growing spine: When should treatment be conservative and when should it be surgical? Orthopade. 2016.45 (6): 518-526.

[16] Zhu X, Wei X, Chen J, et al. Posterior hemivertebra resection and monosegmental fusion in the treatment of congenital scoliosis. Ann R Coll Surg Engl. 2014. 96 (1): 41-44. 